\title{
Pré-éclampsie sévère et hémorragie post-partum: apport de l'échographie « corps entier »
}

\section{Role of whole-body ultrasound in severe pre-eclampsia and post-partum hemorrhage}

\author{
Laurent Zieleskiewicz, MD - Candice Pierrou, MD - Benoit Ragonnet, MD • \\ Maxime Tourret, MD - Cécile Chau, MD - Fabien Craighero, MD • \\ Claire Contargyris, MD - Claude Martin, MD - Marc Leone, MD, PhD
}

Received: 23 August 2012/Accepted: 7 May 2013/Published online: 17 May 2013

(C) Canadian Anesthesiologists' Society 2013

\begin{abstract}
Résumé
Objectif La prise en charge des patientes pré-éclamptiques sévères est un défi pour les équipes travaillant en secteur obstétrical. Nous montrons ici que l'échographie de plusieurs organes du corps réalisée au lit d'une patiente a apporté des informations nécessaires à la prise en charge, sans nécessité de la transférer en service de radiologie ni d'avoir recours à des intervenants extérieurs.
\end{abstract}

Éléments cliniques Une patiente pré-éclamptique sévère de 29 ans avec un HELLP syndrome (hémolyse, cytolyse,

Cet article est accompagné d'un éditorial. Veuillez vous référer à: Can J Anesth 2013; 60: ce numéro.

Contributions des auteurs Maxime Tourret, Cécile Chau, Laurent Zieleskiewicz et Benoit Ragonnet ont évalué la patiente. Laurent Zieleskiewicz et Fabien Craighero ont contribué à l'évaluation échographique de la patiente. Claire Contargyris et Candice Pierrou ont participé à la collecte des données. Marc Leone, Claude Martin et Laurent Zieleskiewicz ont analysé les données et participé à la préparation du manuscrit.

L. Zieleskiewicz, MD · C. Pierrou, MD - B. Ragonnet, MD .

M. Tourret, MD - C. Contargyris, MD · C. Martin, MD .

M. Leone, MD, PhD ( $\square)$

Service d'anesthésie et de réanimation, Hôpital Nord, Assistance

Publique-Hôpitaux de Marseille, Aix Marseille Université,

chemin des Bourrely, 13015 Marseille, France

e-mail: marc.leone@ap-hm.fr

C. Chau, MD

Service de gynécologie obstétrique, Hôpital Nord, Assistance Publique-Hôpitaux de Marseille, Aix Marseille Université,

Marseille, France

F. Craighero, MD

Service de radiologie, Hôpital Nord, Assistance

Publique-Hôpitaux de Marseille,

Aix Marseille Université, Marseille, France thrombopénie) développe en post-partum une hémorragie utérine occulte diagnostiquée grâce à une échographie abdomino-pelvienne réalisée au chevet. L'échoguidage a aussi simplifié la mise en place d'un abord veineux central. Cette patiente évolue favorablement après une hystérectomie d'hémostase et l'administration de facteur VII activé. Les échographies pulmonaire et cardiaque ont optimisé la prise en charge hémodynamique non invasive de cette patiente ayant développé un choc hémorragique puis un xedème pulmonaire. L'échocardiographie a guidé le remplissage vasculaire et l'échographie pulmonaire a permis de détecter l'apparition de l'œè̀me pulmonaire interstitiel et d'en suivre l'évolution.

Conclusion Lespraticiens doivent être informés de l'intérêt de l'échographie du « corps entier »dans le diagnostic et le traitement de pathologies complexes impliquant plusieurs organes, comme la pré-éclampsie. De plus, l'échographie facilite la prise en charge de l'hémodynamie globale. La formation des anesthésiologistes aux différentes techniques échographiques devrait être encouragée.

\footnotetext{
Abstract

Purpose Management of severe pre-eclamptic patients is a challenge for the staff on obstetrical wards. We demonstrate that ultrasound applied to several organs performed at a patient's bedside gave the information required for the patient's management, without the need to transfer her to the radiology department or to call external consultants.

Clinical features A 29-yr-old severely pre-eclamptic patient with HELLP syndrome (hemolysis, cytolysis, thrombopenia) presented, in the post-partum period, with an occult uterine hemorrhage diagnosed with bedside abdominal/pelvic ultrasound. Ultrasound was also used to insert a central venous catheter. After undergoing a
} 
hysterectomy to control hemorrhage and receiving activated factor VII, the patient recovered uneventfully. Hemodynamic management was optimized non-invasively using pulmonary and cardiac ultrasound, when the patient developed hemorrhagic shock followed by pulmonary edema. Volume replacement was guided by cardiac ultrasound findings, and we were able to detect incipient interstitial pulmonary edema and follow its course using pulmonary ultrasound.

Conclusion Practitioners must be aware of the role of whole-body ultrasound in the diagnosis and treatment of complex, multi-organ conditions such as pre-eclampsia. Moreover, ultrasound helps in the management of global hemodynamics. The training of anesthesiologists in a variety of ultrasound techniques should be encouraged.

En obstétrique, certaines situations cliniques comme la pré-éclampsie et le HELLP syndrome (syndrome associant hémolyse, cytolyse et thrombopénie compliquant une pré-éclampsie) nécessitent des prises en charge complexes impliquant plusieurs organes avec la nécessité de prises de décision urgentes. ${ }^{1-3}$ L'examen clinique s'avère souvent difficile et le transport des patientes reste à risque. Nous montrons dans le cas suivant comment l'échographie $\mathrm{du}$ « corps entier » facilite la prise en charge et aide aux décisions thérapeutiques. ${ }^{4}$ La patiente a consenti par écrit à la publication de ce cas.

\section{Éléments cliniques}

Une patiente primipare de 29 ans sans antécédent est admise au service d'obstétrique à 27 semaines d'aménorrhée pour pré-éclampsie. Un traitement antihypertenseur est débuté et des corticoïdes sont administrés pour la maturation fœtale. Quatre jours après l'admission, le bilan biologique évoque un HELLP syndrome: hémoglobine $69 \mathrm{~g} \cdot \mathrm{L}^{-1}$; plaquettes 17 $\mathrm{G} \cdot \mathrm{L}^{-1}$; cytolyse à trois fois la valeur normale. Une extraction fœtale par césarienne est donc décidée collégialement. Une anesthésie générale est réalisée avec induction à séquence rapide en utilisant du thiopenthal $5 \mathrm{mg} \cdot \mathrm{kg}^{-1}$ et de la succinylcholine $1 \mathrm{mg} \cdot \mathrm{kg}^{-1}$. L'intervention se déroule sans incident, la trachée est rapidement extubée en postopératoire. Trois culots globulaires sont transfusés. L'hémoglobinémie postopératoire est de $12 \mathrm{~g} \cdot \mathrm{L}^{-1}$. Dans les heures qui suivent, la patiente reste anurique malgré un remplissage de $4 \mathrm{~L}$ de cristalloïdes. Sept heures après la césarienne, le bilan montre une insuffisance rénale (créatinine à $140 \mu \mathrm{mol} \cdot \mathrm{L}^{-1}$; urée à $13 \mathrm{mmol} \cdot \mathrm{L}^{-1}$ ) avec une hyperkaliémie à $6,6 \mathrm{mmol} \cdot \mathrm{L}^{-1}$ associée à une coagulation intravasculaire disséminée (plaquettes 17
$\mathrm{G} \cdot \mathrm{L}^{-1}$; taux de prothrombine (TP) $58 \%$; fibrinogène $1,2 \mathrm{~g} \cdot \mathrm{L}^{-1}$; D-Dimères $16 \mu \mathrm{g} \cdot \mathrm{mL}^{-1}$ et complexes solubles > $\left.150 \mu \mathrm{g} \cdot \mathrm{L}^{-1}\right)$. Devant ces troubles métaboliques, la patiente est transférée de la salle de réveil aux soins intensifs pour hémodiafiltration.

Dans ce contexte de trouble de l'hémostase, la mise en place du cathéter de dialyse triple lumière dans la veine jugulaire interne droite est réalisée sous échographie (Philips CX 50 compactXtreme ultrasound system ${ }^{\mathrm{TM}}$; Philips Medical Systems, Suresnes, France) en utilisant une sonde linéaire vasculaire (Philips L12-3 ${ }^{\mathrm{TM}}$ ). La patiente est dialysée à cause de l'anurie et l'hyperkaliémie. Sur le plan hémodynamique, elle est hypertendue (tension artérielle: $160 / 90 \mathrm{mmHg}$ ). Cliniquement, aucun saignement n'est extériorisé, ni spontanément, ni à l'expression utérine. La thrombopénie est initialement attribuée au HELLP syndrome. Par conséquent, aucune transfusion plaquettaire n'est effectuée.

À l'échocardiographie transthoracique (ETT) réalisée à son admission aux soins intensifs, les pressions de remplissage s'avèrent non élevées. En coupe quatre cavités, l'analyse du flux mitral donne un rapport E/ $\mathrm{A}=1,2$ en Doppler pulsé et un rapport $\mathrm{E} / \mathrm{E}$ ' à 6 en Doppler tissulaire. ${ }^{5}$ En coupe apicale cinq cavités, l'intégrale temps-vitesse aortique est augmentée de plus de $13 \%$ après épreuve de lever de jambe passif. ${ }^{6}$ Une dépendance à la précharge est donc diagnostiquée, ce qui suggère une hypovolémie.

À l'échographie pulmonaire réalisée dans le même temps, aucun épanchement pleural n'est visualisé. Au niveau parenchymateux antérieur, on note un profil normal avec glissement pleural et lignes A horizontales (artéfacts de répétition de la ligne pleurale). Nous ne retrouvons pas de lignes B verticales (lignes de Kerley échographiques). La présence d'un œdème pulmonaire interstitiel est donc exclue. ${ }^{7}$ Ces trouvailles justifient la poursuite de l'expansion volémique par l'administration de $3 \mathrm{~L}$ de cristalloïdes. À cause d'une hypoalbuminémie à $13 \mathrm{~g} \cdot \mathrm{L}^{-1}$ et d'une protéinurie $>12 \mathrm{~g} \cdot \mathrm{j}^{-1}$, la patiente reçoit également l'administration fractionnée de $800 \mathrm{~mL}$ d'albumine à 20 $\%$, soit $160 \mathrm{~g}$.

Six heures après son admission aux soins intensifs, l'hémoglobinémie passe à $40 \mathrm{~g} \cdot \mathrm{L}^{-1}$, la thrombopénie demeure persistante à $14 \mathrm{G} \cdot \mathrm{L}^{-1}$ et le TP chute à $34 \%$. Cette anémie aigue est attribuée à l'hémolyse, une hémorragie occulte ou une hémodilution. À l'examen obstétrical, on retrouve toujours l'absence de saignement à l'expression utérine mais une augmentation de la hauteur utérine de $3 \mathrm{~cm}$ par rapport à l'ombilic.

L'échographie pelvienne permet alors la mise en évidence d'un volumineux hématome utérin (figures 1A et 1B) sans signal Doppler couleur ou pulsé, ce qui exclut une étiologie vasculaire (anévrisme par exemple). ${ }^{8}$ Cet 
hématome est évalué à $12,6 \mathrm{~cm}$ de hauteur, $14,4 \mathrm{~cm}$ de profondeur et $12 \mathrm{~cm}$ de largeur; son volume est estimé à environ $1,5 \mathrm{~L} .{ }^{9}$

À l'échographie abdominale, il y a absence d'hémorragie intrapéritonéale ou des flancs, ce qui exclut un hématome rétro-capsulaire du foie ou une rupture vasculaire utéro-ovarienne. ${ }^{10,11} \mathrm{~L}$ 'origine intra-utérine du saignement est confirmée. L'hémodynamie est conservée avec une tension artérielle à $134 / 84 \mathrm{mmHg}$ et une fréquence cardiaque à 130 battements par min. Cependant, devant les données échographiques et la tachycardie, l'indication d'hémostase chirurgicale en urgence au niveau utérin est décidée collégialement. La patiente reçoit une transfusion de trois concentrés de globules rouges, de trois unités de plasma frais congelés et d'une unité plaquettaire. Une mesure de la pression intravésicale à $20 \mathrm{mmHg}$ conduit au diagnostic du syndrome du compartiment abdominal. L'état respiratoire se dégrade rapidement, nécessitant une intubation orotrachéale en séquence rapide en utilisant la kétamine

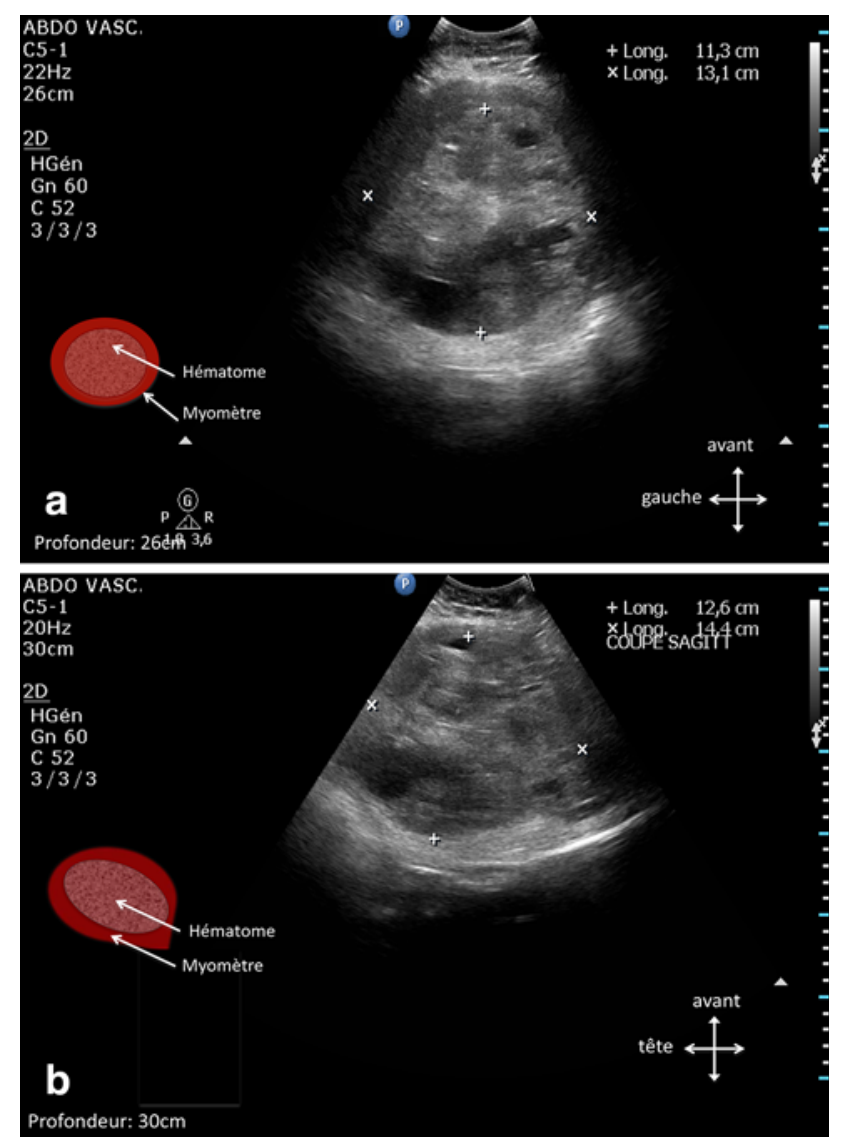

Fig. 1 a Coupe échographique pelvienne transverse (sonde abdominale convexe 5 à $1 \mathrm{MHz}$ ) de l'hématome intra-utérin dont le volume est évalué à plus de $1,5 \mathrm{~L}$. b Coupe échographique pelvienne sagittale (sonde abdominale convexe 5 à $1 \mathrm{MHz}$ ) montrant un volumineux hématome intra-utérin. A noter l'absence de flux Doppler en son sein et le rocuronium. Une échographie cardiopulmonaire est réalisée en quelques minutes pendant la transfusion des produits dérivés sanguins et la préparation de la patiente.

À l'échographie pulmonaire, nous mettons en évidence des lignes verticales multiples, mobiles avec la plèvre, bien définies jusqu'au bas de l'écran sans épuisement. Ces lignes B antérieures bilatérales symétriques sont des artéfacts de répétition liés à la réverbération des ultrasons dans les septas interstitiels épaissis. ${ }^{7}$ Elles dénotent la présence d'un syndrome interstitiel aigu, i.e., un œdème pulmonaire dans ce contexte (figure 2). ${ }^{7}$ À l'ETT réalisée dans le même temps, les pressions de remplissage apparaissent normales: en coupe quatre cavités avec analyse du flux mitral, nous retrouvons un rapport E/ $\mathrm{A}=1$ en Doppler pulsé et un rapport E/E' à 7 en Doppler tissulaire. ${ }^{5}$ Par conséquent, le remplissage vasculaire est limité à l'administration de produits dérivés sanguins supplémentaires (six culots globulaires, six unités de plasma frais et une unité plaquettaire). Par la suite, les échanges gazeux s'améliorent partiellement suite à l'installation d'une pression expiratoire positive à $12 \mathrm{mmHg}$, ce qui permet le transfert de la patiente au bloc opératoire $40 \mathrm{~min}$ après la décision d'opérer.

Une laparotomie par la cicatrice de césarienne est alors réalisée immédiatement sous anesthésie générale au midazolam, sufentanil et cisatracurium. Un hématome intra-utérin, quantifié à $2 \mathrm{~L}$, est évacué. On note une ischémie utérine majeure et un saignement diffus. Devant l'inefficacité des massages utérins, des utérotoniques, du fibrinogène $(1,5 \mathrm{~g})$, de l'acide tranexamique $(1 \mathrm{~g}$ en une heure puis $4 \mathrm{~g}$ par $24 \mathrm{~h}$ ) et d'une troisième unité plaquettaire, une hystérectomie est réalisée. Ensuite, devant une coagulopathie clinique et biologique du facteur VII activé $\left(90 \mu \mathrm{g} \cdot \mathrm{kg}^{-1}\right)$ est prescrit. Le saignement cède dans les minutes qui suivent la première

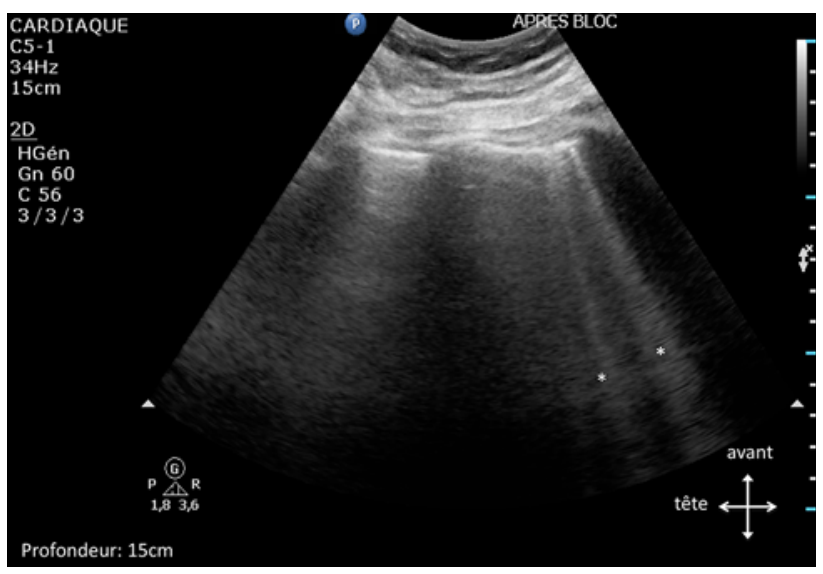

Fig. 2 Coupe échographique pulmonaire longitudinale antérieure (sonde abdominale convexe 5 à $1 \mathrm{MHz}$ ) montrant la présence de lignes $\mathrm{B}$ signant un œdème pulmonaire 
administration. À son retour aux soins intensifs, la patiente est hémodynamiquement stable sans catécholamines: pression artérielle à 132/92 $\mathrm{mmHg}$, pouls à 92 battements par min. Il persiste une défaillance respiratoire avec un rapport $\mathrm{PaO}_{2} / \mathrm{F}_{\mathrm{I}} \mathrm{O}_{2}$ à 100 chez une patiente en ventilation assistée contrôlée avec une pression expiratoire positive à $12 \mathrm{cmH}_{2} \mathrm{O}$.

A l'ETT (figures 3 et 4), nous retrouvons maintenant des pressions de remplissage élevées (rapport $\mathrm{E} / \mathrm{A}>2$; $\left.\mathrm{E} / \mathrm{E}^{\prime}=9,75\right)$ et l'absence de critère de précharge-dépendance (absence de variation respiratoire de la veine cave inférieure, absence d'augmentation de l'intégrale temps vitesse aortique au lever de jambe). ${ }^{5,6} \mathrm{La}$ fraction d'éjection du ventricule gauche est évaluée à $45 \%$. A l'échographie pulmonaire, on note des lignes B diffuses évoquant un

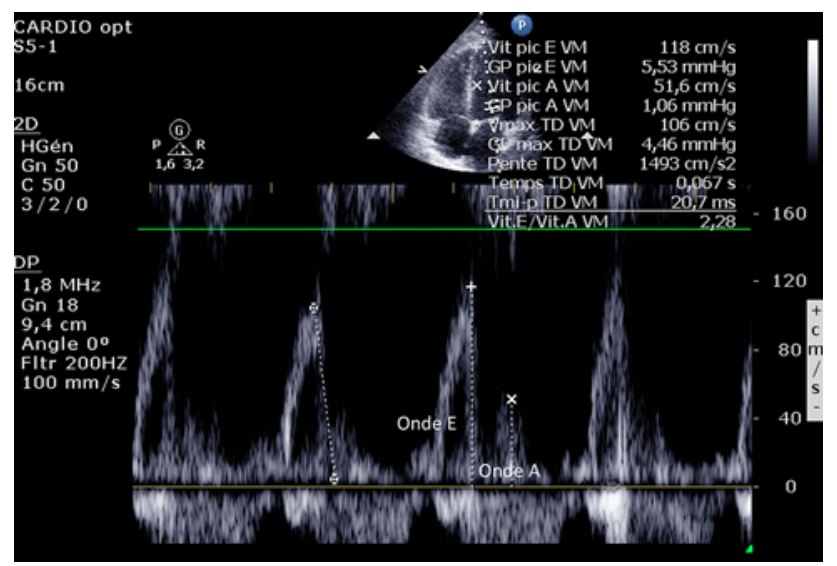

Fig. 3 Échographie cardiaque en coupe apicale quatre cavités (sonde cardiaque sectorielle 2 à $4 \mathrm{MHz}$ ): doppler du flux mitral identifiant un rapport E/A à 2,28 et un temps de décroissance de l'onde $\mathrm{E}$ court en faveur de pressions de remplissage élevées. Ceci est en faveur d'une participation cardiaque à l'œdème pulmonaire

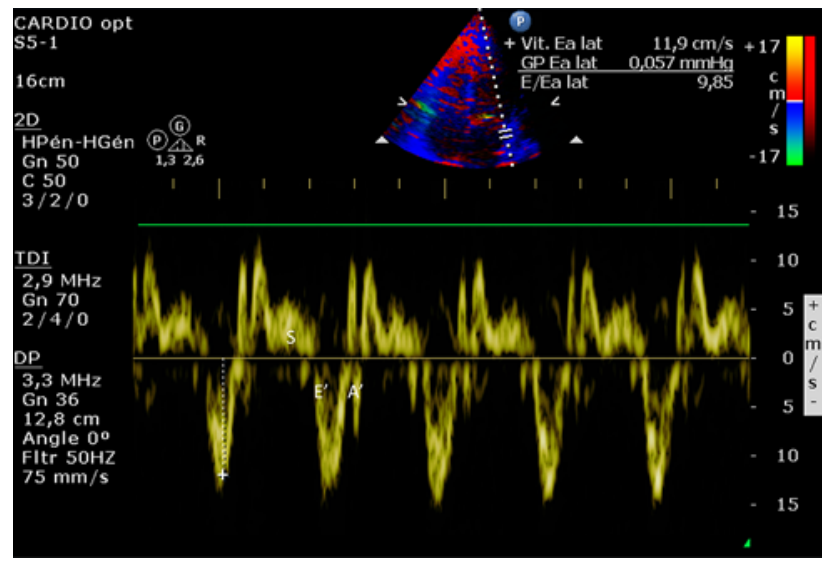

Fig. 4 Échographie cardiaque en coupe apicale quatre cavités: Doppler tissulaire de l'anneau mitral latéral (sonde cardiaque sectorielle 2 à $4 \mathrm{Mhz}$ ). Rapport E/E' à 9.85 en faveur de pressions de remplissage plus élevées œdème pulmonaire alvéolo-interstitiel (figure 2). ${ }^{7}$ Devant cette défaillance respiratoire avec des pressions de remplissage élevées, on administre du furosémide $20 \mathrm{mg}$, ce qui augmente la diurèse augmente et améliore l'hématose. La trachée de la patiente est extubée moins de $48 \mathrm{~h}$ plus tard.

Quatre jours après son admission, la patiente sort des soins intensifs. Elle est eupnéique à l'air ambiant. Une hypertension persiste, traitée par de la nicardipine. La créatinine plasmatique est à $346 \mu \mathrm{mol} \cdot \mathrm{L}^{-1}$ mais la diurèse est conservée. L'hémoglobine reste stable à $90 \mathrm{~g} \cdot \mathrm{L}^{-1}$, les plaquettes à $56 \mathrm{G} \cdot \mathrm{L}^{-1}$. La patiente retourne à son domicile 23 jours après l'accouchement. L'examen clinique et le bilan biologique sont normalisés: hémoglobinémie $98 \mathrm{~g} \cdot \mathrm{L}^{-1}$, plaquettes $310 \mathrm{G} \cdot \mathrm{L}^{-1}$ et créatininémie $95 \mu \mathrm{mol} \cdot \mathrm{L}^{-1}$.

\section{Discussion}

Ce cas clinique illustre l'intérêt de l'échographie corps entier dans la prise en charge de l'état hémodynamique d'une patiente altéré du fait d'une pré-éclampsie sévère et d'un choc hémorragique (Tableau). Initialement cantonnée à l'exploration cardiographique, l'utilisation des ultrasons en anesthésiologie connait un développement important avec l'exploration pleuro-pulmonaire, abdominale et vasculaire (échographie générale), voire cérébrale. ${ }^{4,12} \mathrm{Ce}$ type d'examen multisite est dénommé échographie « corps entier» ou plus récemment échographie «head to toe » (« de la tête aux pieds »). ${ }^{4,13}$ Par analogie à la tomodensitométrie du corps entier, certains auteurs l'utilisent de manière systématique chez les patients instables à l'admission. ${ }^{13}$ Ceci modifie le diagnostic dans $25 \%$ des cas. ${ }^{13}$ Dans notre cas, l'échographie corps entier était réalisée de manière ciblée, en fonction des points d'appels pour répondre à des questions cliniques précises (Tableau).

L'ensemble des examens a été réalisé avec une seule machine d'échographie équipée de trois sondes (abdominale convexe C5-1, cardiaque sectorielle S 5-1, et linéaire L 12-3). Seule l'échographie cardiaque nécessite une sonde spécifique. Les échographies abdominale, pulmonaire et vasculaire sont réalisées avec des sondes et appareils classiquement utilisés par les obstétriciens et les anesthésiologistes (anesthésie loco-régionale). ${ }^{14}$ Elles sont la plupart du temps disponibles dans les maternités. On note que l'échographie abdominale, pulmonaire et cardiaque fait actuellement partie des compétences requises pour les réanimateurs. ${ }^{12}$ Enfin, l'intérêt et la faisabilité du Doppler transcrânien et de l'évaluation échographique $\mathrm{du}$ diamètre $\mathrm{du}$ nerf optique ont été récemment démontrés chez les parturientes pré-éclamptiques. ${ }^{15}$ 


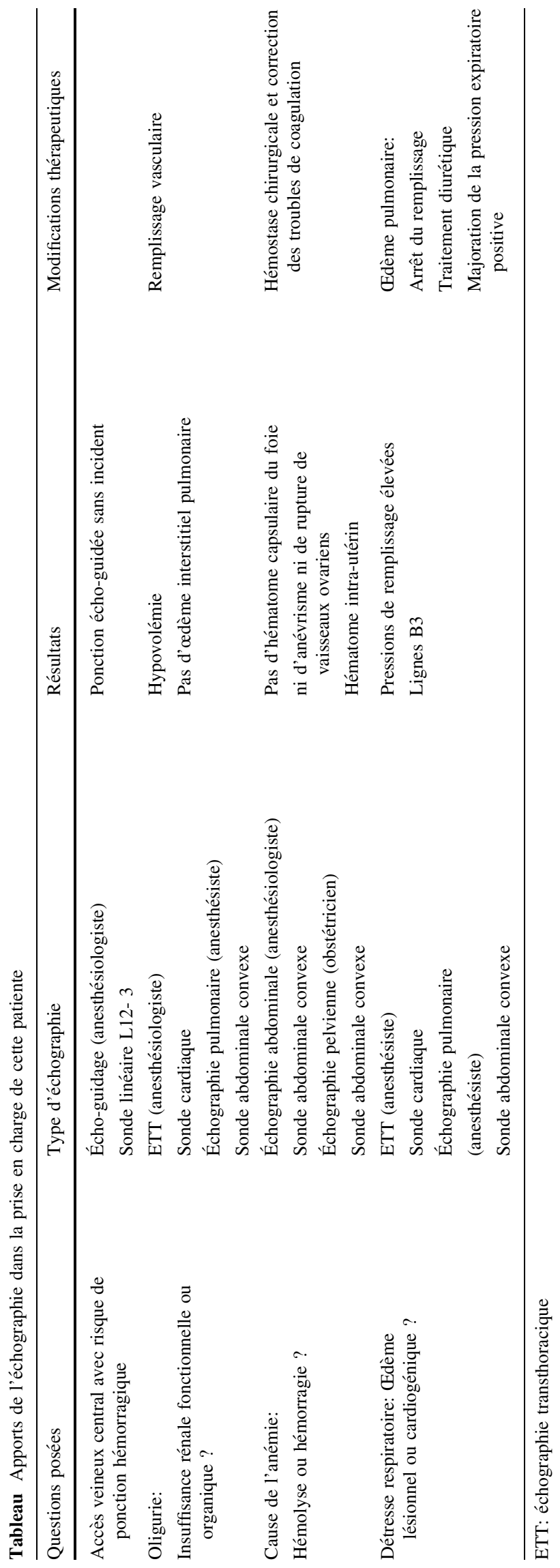

L'échographie abdominopelvienne facilite le diagnostic des hémorragies occultes du post-partum. Sa sensibilité est supérieure à l'examen clinique et à l'expression utérine. ${ }^{16}$ Chez notre patiente, l'examen clinique ne démontrait pas de saignement. L'intérêt de l'échographie pelvienne est majeur en cas d'hémorragie survenant après une césarienne. ${ }^{16}$ Les informations qu'elle apporte sont multiples. Dans une étude réalisée chez 125 patientes ayant une hémorragie de la délivrance, la présence d'une masse intra-utérine ou d'un épanchement intra-abdominal était associée à un mauvais pronostic. ${ }^{17}$ Un signal Doppler couleur ou pulsé au sein de l'hématome évoque une rupture d'un pseudoanévrisme des artères utérines. ${ }^{8}$ Le calcul du volume de l'hématome est automatisé sur la plupart des machines. Dans notre observation, la détection d'un hématome volumineux a permis de poser le diagnostic d'hémorragie de préférence à l'hémolyse pour expliquer l'anémie, ce qui a accéléré la prise en charge chirurgicale. Enfin, l'échographie abdominopelvienne élimine d'autres causes d'hémorragie du péripartum comme un hématome sous capsulaire du foie ou une rupture spontanée des vaisseaux utéro-ovariens. ${ }^{10,11}$

L'échographie est utile dans les situations hémodynamiques complexes. La pré-éclampsie est une situation à risque d'œedème pulmonaire et d'insuffisance rénale. ${ }^{3}$ Par ailleurs, le choc hémorragique impose une expansion volémique et la transfusion de produits dérivés sanguins en urgence. Toutefois, le monitorage invasif de ces patientes par cathétérisme artériel pulmonaire n'est plus recommandé. ${ }^{18}$ L'utilisation de l'échocardiographie est par contre fortement conseillée. ${ }^{19}$ L'utilisation de marqueurs dynamiques prédictifs de réponse au remplissage évite toute expansion volémique inutile, notamment chez des parturientes à risque d'œdème pulmonaire. En effet, la variation de l'intégrale temps-vitesse aortique après épreuve de lever de jambe est un critère non invasif qui a été validé chez les patients en ventilation spontanée $^{6}$ et chez 25 patientes pré-éclamptiques sévères. ${ }^{20}$ L'utilisation de ce paramètre chez notre patiente a conduit à la poursuite de l'expansion volémique, tant que la patiente était en situation de précharge dépendance. L'échocardiographie évalue également les pressions de remplissage du ventricule gauche et sa relaxation estime donc le risque d'œdème aigu du poumon. ${ }^{5}$ En fait, la physiopathologie de l'œdème pulmonaire en péripartum est multifactorielle et une augmentation des pressions de remplissage n'est pas systématique. $^{21}$ L'échographie pulmonaire détectant l'œdème interstitiel à un stade préclinique, tout remplissage vasculaire délétère pourrait être arrêté avant la survenue de signes cliniques et d'un œè̀me alvéolaire. $^{7,22}$ Nous avons rapporté son utilisation pour 
optimiser la prise en charge hémodynamique des patientes pré-éclamptiques sévères et des parturientes dyspnéiques. ${ }^{23}$ Dans le cas présent, le profil échographique pulmonaire était initialement normal. Plus tard, il était en faveur d'un œdème pulmonaire, conduisant à l'arrêt du remplissage vasculaire puis à l'instauration d'un traitement diurétique. Cette prise en charge a été associée à la normalisation de l'hématose en quelques heures, permettant une extubation trachéale rapide.

La principale limite de l'échographie est son degré de dépendance à l'opérateur. Dans notre cas, l'anesthésiologiste en charge de la patiente est enseignant pour un diplôme universitaire d'exploration ultrasonographique aux soins intensifs. Il est certifié et pratique l'échographie depuis cinq ans. Cependant, si une formation avancée en échocardiographie nécessite un an et de 150 à 200 examens, la formation de base à l'échographie générale est plus courte et facilement généralisable (environ $10 \mathrm{~h}$ de cours et 30 examens recommandés). ${ }^{12}$ Elle donne des compétences en échographie pleuropulmonaire, abdominale et vasculaire. Une étude démontre qu'une formation accélérée d'une heure permet d'obtenir un niveau de compétence acceptable dans la détection échographique de l'œdème pulmonaire. ${ }^{24}$ Ainsi, même un opérateur moins formé peut tirer un grand nombre d'informations de l'échographie corps entier. En cas de doute sur des résultats, le recours à un intervenant extérieur est une solution satisfaisante en fonction de leurs disponibilités. Dans ce cas clinique, seule l'échographie pelvienne était réalisée par un non-anesthésiologiste.

En conclusion, l'échographie du « corps entier » rendue possible par la polyvalence de la machine, des sondes utilisées et de l'opérateur a apporté des informations pertinentes modifiant pas à pas la prise en charge. Son utilisation ciblée répond à un grand nombre de situations cliniques complexes. Nous pensons que l'ensemble des anesthésiologistes prenant en charge des patientes pré-éclamptiques sévères devrait être informé de l'intérêt majeur de l'échographie, examen non invasif, réalisé au lit de la patiente, sans nécessité absolue de faire appel à un correspondant supplémentaire. Ainsi, la formation des anesthésiologistes à l'échographie du « corps entier » devrait être poursuivie selon le même modèle qu'aux soins intensifs. ${ }^{25}$

Conflit d'intérêts Aucun.

\section{Références}

1. Sibai BM, Ramadan MK, Usta I, Salama M, Mercer BM, Friedman SA. Maternal morbidity and mortality in 442 pregnancies with hemolysis, elevated liver enzymes, and low platelets (HELLP syndrome). Am J Obstet Gynecol 1993; 169: 1000-6.

2. Barton JR, Sibai BM. Acute life-threatening emergencies in preeclampsia-eclampsia. Clin Obstet Gynecol 1992; 35: 402-13.

3. Young $P$, Johanson $R$. Haemodynamic, invasive and echocardiographic monitoring in the hypertensive parturient. Best Pract Res Clin Obstet Gynaecol 2001; 15: 605-22.

4. Karabinis A, Fragou M, Karakitsos D. Whole-body ultrasound in the intensive care unit: a new role for an aged technique. J Crit Care 2010; 25: 509-13.

5. Nagueh SF, Middleton KJ, Kopelen HA, Zoghbi WA, Quinones $M A$. Doppler tissue imaging: a noninvasive technique for evaluation of left ventricular relaxation and estimation of filling pressures. J Am Coll Cardiol 1997; 30: 1527-33.

6. Lamia B, Ochagavia A, Monnet X, Chemla D, Richard C, Teboul $J L$. Echocardiographic prediction of volume responsiveness in critically ill patients with spontaneously breathing activity. Intensive Care Med 2007; 33: 1125-32.

7. Lichtenstein D, Meziere G, Biderman P, Gepner A, Barre $O$. The comet tail artifact. An ultrasound sign of alveolar-interstitial syndrome. Am J Respir Crit Care Med 1997; 156: 1640-6.

8. McGonegle SJ, Dziedzic TS, Thomas J, Hertzberg BS. Pseudoaneurysm of the uterine artery after an uncomplicated spontaneous vaginal delivery. J Ultrasound Med 2006; 25: 1593.

9. Dicuio M, Pomara G, Menchini Fabris F, Ales V, Dahlstrand C, Morelli $G$. Measurements of urinary bladder volume: comparison of five ultrasound calculation methods in volunteers. Arch Ital Urol Androl 2005; 77: 60-2.

10. Kinthala S, Fakoory M, Greaves T, Kandamaran L, Thomas H, Moe $W$. Subcapsular liver hematoma causing cardiac tamponade in HELLP syndrome. Int J Obstet Anesth 2012; 21: 276-9.

11. Ziereisen V, Bellens B, Gerard C, Baeyens L. Spontaneous rupture of utero-ovarian vessels in postpartal period: a case report and review of the literature (French). J Gynecol Obstet Biol Reprod (Paris) 2003; 32: 51-4.

12. Mayo PH, Beaulieu Y, Doelken P, et al. American College of Chest Physicians/La Société de Réanimation de Langue Française statement on competence in critical care ultrasonography. Chest 2009; 135: 1050-60.

13. Manno E, Navarra M, Faccio L, et al. Deep impact of ultrasound in the intensive care unit: the "ICU-sound" protocol. Anesthesiology 2012; 117: 801-9.

14. Arbelot $C$, Ferrari F, Bouhemad B, Rouby JJ. Lung ultrasound in acute respiratory distress syndrome and acute lung injury. Curr Opin Crit Care 2008; 14: 70-4.

15. Dubost C, Le Gouez A, Jouffroy V, et al. Optic nerve sheath diameter used as ultrasonographic assessment of the incidence of raised intracranial pressure in preeclampsia: a pilot study. Anesthesiology 2012; 116: 1066-71.

16. Shalev J, Royburt M, Fite G, et al. Sonographic evaluation of the puerperal uterus: correlation with manual examination. Gynecol Obstet Invest 2002; 53: 38-41.

17. Lousquy R, Morel O, Soyer P, Malartic C, Gayat E, Barranger E. Routine use of abdominopelvic ultrasonography in severe postpartum hemorrhage: retrospective evaluation in 125 patients. Am J Obstet Gynecol 2011; 204: 232.e1-6.

18. Li YH, Novikova N. Pulmonary artery flow catheters for directing management in pre-eclampsia. Cochrane Database Syst Rev 2012; 6: 8882 .

19. Dennis AT. Management of pre-eclampsia: issues for anaesthetists. Anaesthesia 2012; 67: 1009-20.

20. Brun C, Zieleskiewicz L, Textoris J, et al. Prediction of fluid responsiveness in severe preeclamptic patients with oliguria. Intensive Care Med 2013; 39: 593-600.

21. Dennis AT, Solnordal CB. Acute pulmonary oedema in pregnant women. Anaesthesia 2012; 67: 646-59. 
22. Lichtenstein D, Karakitsos D. Integrating lung ultrasound in the hemodynamic evaluation of acute circulatory failure (the fluid administration limited by lung sonography protocol). J Crit Care 2012; 27: 533.e11-9.

23. Zieleskiewicz L, Lagier D, Contargyris C, et al. Lung ultrasoundguided management of acute breathlessness during pregnancy. Anaesthesia 2013; 68: 97-101.
24. Noble VE, Lamhaut L, Capp R, et al. Evaluation of a thoracic ultrasound training module for the detection of pneumothorax and pulmonary edema by prehospital physician care providers. BMC Med Educ 2009; 9: 3.

25. Expert Round Table on Ultrasound in ICU. International expert statement on training standards for critical care ultrasonography. Intensive Care Med 2011; 37: 1077-83. 